\title{
TENSOATIVIDADE DE ÁCIDOS HÚMICOS DE PROCEDÊNCIAS DISTINTAS
}

Thalita Grando Rauen, Nito Ângelo Debacher e Maria Marta de Souza Sierra*

Departamento de Química, Universidade Federal de Santa Catarina, CP 476, 88040-900 Florianópolis - SC

Eduardo Juan Soriano Sierra

Núcleo de Estudos do Mar, Universidade Federal de Santa Catarina, CP 476, 88040-900 Florianópolis - SC

Recebido em 2/7/01; aceito em 22/1/02

\begin{abstract}
TENSOACTIVITY OF HUMIC ACIDS FROM DISTINCT ENVIRONMENTS. The surface activity of humic acids (HA) is a parameter which might be considered in the evaluation of all types of aggregation of these substances. In this work the surface tension of aquatic and terrestrial-HA aqueous solutions was observed under varied conditions of $\mathrm{pH}$, concentration and ionic strength. Results showed that HA present surface activity for a extent range of concentration. The surface tension decreases with HA concentration increasing and, for all samples exist a concentration value above which a reduction in the rate of the solute migration to the solution surface is observed. This value is sensibly reduced and sharpened with the increasing of the ionic strength. Surface tension is also reduced with the acidity increasing, being a minimum reached between $\mathrm{pH} 3$ and 5. Observed effects are explained in view of conformational arrangements of HA which tend to agglomerate in micelle-like domains in aqueous solutions.
\end{abstract}

Keywords: humic acids; surface tension; micelar model.

\section{INTRODUÇÃO}

Substâncias húmicas (SH) são produtos da degradação oxidativa e subsequiente polimerização da matéria orgânica animal e vegetal. Elas são constituídas por uma mistura de compostos de elevada massa molar com uma grande variedade de grupos funcionais. Suas características moleculares podem variar dependendo da idade ou da origem do material sendo, por isso, definidas operacionalmente. Assim, com base nas suas respectivas solubilidades são classificadas em: humina (insolúvel em meio aquoso), ácidos fúlvicos (solúveis em água em qualquer $\mathrm{pH}$ ) e ácidos húmicos (solúveis em água em pHs alcalinos).

Como representantes majoritários do carbono orgânico em solos, sedimentos e águas estas substâncias têm profunda influência nos ciclos biogeoquímicos de constituintes naturais e antropogênicos nestes ambientes. Elas podem, por exemplo, atuar como receptores de elétrons na respiração microbiana, estimulando a redução de metais ${ }^{1}$, ou ainda, na bioconcentração de micropoluentes orgânicos em solução aquosa ${ }^{2}$. Em certas situações, suas características funcionais têm sido inclusive utilizadas como indicadoras do grau de poluição em diferentes ambientes ${ }^{3}$. Assim, o conhecimento das propriedades físico-químicas deste material, assim como de suas interações com xenobióticos, são aspectos fundamentais que devem ser relevados no estudo da recuperação e manejo de ecossistemas impactados. As dificuldades inerentes destes estudos originam-se, principalmente, nas diferenças estruturais entre SH de diferentes origens e, ainda, na riqueza de peculiaridades estruturais em cada uma delas.

Atualmente, busca-se a correlação entre os parâmetros estruturais e a capacidade de interação das SH com as diferentes famílias de poluentes metálicos e orgânicos ${ }^{2,4}$. Entretanto, se por um lado, os estudos das interações SH-metais produziram um número considerável de dados convergentes na literatura, as propostas a respeito dos

\footnotetext{
*e-mail: sierra@qmc.ufsc.br
}

mecanismos envolvendo as interações SH-compostos orgânicos têm sido bastante controversas. Estudos envolvendo complexação ${ }^{5}$, adsorção ${ }^{6}$, oclusão nos espaços vazios das moléculas ${ }^{7}$ e partição em microambientes hidrófóbos ${ }^{8}$ são algumas das possibilidades consideradas. Recentemente, Wandruszka e colaboradores sugeriram que o modo de interação entre SH e espécies hidrófobas de massa molar reduzida é similar àquele observado para surfactantes ${ }^{9-12}$. Segundo estes dados, devido ao seu caráter anfifílico as $\mathrm{SH}$, principalmente os ácidos húmicos (AH), podem agregar-se inter e/ou intramolecularmente, em solução aquosa, produzindo aglomerados pseudo-micelares capazes de sequiestrar espécies hidrófobas.

Apesar das características anfifílicas das $\mathrm{SH}$ serem relativamente bem documentadas e da tensão superficial (TS) ser um parâmetro relevante para elucidar os tipos de agregações de surfactantes em solução, poucos dados têm sido publicados no que se refere a esta técnica aplicada às SH. A esse respeito pode-se citar os trabalhos de Tchapeck e Wasowski ${ }^{13}$, Chen e Schnitzer ${ }^{14}$, Hayase e Tsubota ${ }^{15}$ e Anderson et $a l .{ }^{16}$ onde foram, em geral, observadas reduções da TS com o aumento da concentração de substâncias húmicas. Mais recentemente, Yates e Wandruszka ${ }^{17}$ mostraram a influência do $\mathrm{pH}$ e da concentração de íons metálicos na TS de soluções de AH e de ácidos fúlvicos. Some-se à escassez de dados o fato de muitos destes experimentos terem sido realizados em condições extremas de concentração e $\mathrm{pH}$, dificultando a extrapolação das interpretações para sistemas naturais. Uma outra limitação refere-se à dificuldade de comparação entre as distintas informações, em virtude da diversidade de metodologias usadas para a obtenção do material estudado.

No presente estudo observamos as variações da TS de soluções aquosas de $\mathrm{AH}$ gerados em ambientes aquáticos e terrestres em função da concentração, do $\mathrm{pH}$ e da força iônica do meio. Os AH foram extraídos e purificados segundo a metodologia recomendada pela IHSS (Sociedade Internacional das Substâncias Húmicas) e as condições físico-químicas dos experimentos foram determinadas de forma a preservar a validade ambiental das interpretações. As informações aqui obtidas servirão de subsídios para estudos das interações entre AH e poluentes orgânicos. 


\section{PARTE EXPERIMENTAL}

\section{Origem, extração e caracterização dos AH}

Os ácidos húmicos foram extraídos, segundo a metodologia sugerida pela IHSS ${ }^{18}$, dos seguintes locais: Lagoa do Peri (AH1); Manguezal de Itacorubí (ITA3) e Manguezal de Ratones (Gran2 e CLA1), todos na Ilha de Santa Catarina. A Lagoa do Peri é um lago costeiro de água doce que será futuramente utilizado para captação e beneficiamento de água potável da região sul da Ilha de Santa Catarina. Os manguezais de Itacorubi e Ratones localizam-se sobre a Baía Norte da Ilha, e têm características geológicas e florísticas equivalentes. Entretanto, enquanto o primeiro encontra-se em meio à maior aglomeração urbana da Ilha, determinando que seja um ambiente fortemente impactado pelas ações antrópicas, o manguezal de Ratones por se localizar em área escassamente povoada, dentro de uma estação ecológica, conserva suas características originais.

$\mathrm{O}$ AH Aldrich, representando um ácido húmico de origem terrestre, foi submetido a um processo de purificação, para redução do teor de cinzas, que consistiu nos seguintes passos: i) re-suspensão de $2 \mathrm{~g}$ da amostra comercial em $\mathrm{HCl} / \mathrm{HF}\left(0,1 / 0,3 \mathrm{~mol} \mathrm{~L}^{-1}\right)$, para dissolução de silicatos, seguida de filtração; ii) lavagem do filtrado com água Milli-Q, até teste negativo para íons cloreto, usando solução de $\mathrm{AgNO}_{3}$; iii) re-dissolução do filtrado em $\mathrm{NaOH} 0,1 \mathrm{~mol} \mathrm{~L}^{-1}$ seguida de eluição através da resina de troca iônica IRA 200, para conversão dos compostos húmicos na forma ácida. A secagem foi feita por liofilização (Edwards Micromodulyo 1.5K Freeze Dryer).

A análise elementar (CHN e S) dos AH foi realizada em um Analisador Elementar Perkin Elmer modelo 2400.

\section{Medidas da tensão superficial (TS) das soluções}

As medidas de TS foram realizadas num tensiômetro Krüss, modelo K8, pelo método do anel (Pt-Ir, $20 \mathrm{~mm}$ de diâmetro) ${ }^{19}$ com as amostras termostatizadas a $22{ }^{\circ} \mathrm{C}$.

Soluções estoque de $\mathrm{AH}$ foram preparadas na concentração de $2000 \mathrm{mg} \mathrm{L}^{-1}$, colocando-se a quantidade necessária de AH num balão volumétrico, adicionando água (sistema Milli-Q, condutividade $<0,1 \mu \mathrm{S} \mathrm{cm}^{-1}$ ) até $50 \%$ do volume do balão, ajustando o $\mathrm{pH}$ em 7 com uma solução de $\mathrm{KOH} 3 \mathrm{~mol} \mathrm{~L}^{-1}$, submetendo as soluções ao ultra-som por $1 \mathrm{~h}$ e completando o volume com água. Estas soluções foram posteriormente filtradas em filtros de microfibra de vidro (GF/C, $47 \mathrm{~mm}$ Watman) previamente calcinados $\left(500^{\circ} \mathrm{C}\right)$ durante $4 \mathrm{~h}$.

Para as medidas da TS em função da concentração, foram usados volumes de $10 \mathrm{~mL}$ das diluições correspondentes.

$\mathrm{Na}$ avaliação do efeito do $\mathrm{pH}$, o procedimento foi como segue: foram preparados $50 \mathrm{~mL}$ de soluções $\left(30 \mathrm{mg} \mathrm{L}^{-1}\right)$ de AH. Cada uma das soluções foi subdividida em duas porções de $25 \mathrm{~mL}$, sendo uma das porções acidificada $(\mathrm{HCl})$ para as medidas em $\mathrm{pH}<7$ e a outra alcalinificada $(\mathrm{KOH})$ para as medidas em $\mathrm{pH}>7$.

Para avaliar o efeito da adição de $\mathrm{NaCl}$, as diluições foram feitas usando-se, no lugar de água destilada, uma solução aquosa $\left(0,3 \mathrm{~mol} \mathrm{~L}^{-1}\right)$ de $\mathrm{NaCl}$. Nestes casos, as soluções foram vigorosamente agitadas e então deixadas em repouso durante pelo menos duas horas antes de serem feitas as medidas de TS.

Todas as medidas foram feitas em triplicata.

\section{RESULTADOS E DISCUSSÃO}

\section{Características gerais dos ácidos húmicos estudados}

As SH são compostas essencialmente de carbono, hidrogênio, nitrogênio, oxigênio, fósforo e enxofre, sendo o carbono e o oxigê- nio os elementos mais abundantes em termos de massa, seguidos de hidrogênio e nitrogênio. O conteúdo de enxofre varia desde quantidades traço até algumas unidades por cento, dependendo da origem das amostras, e o conteúdo em fósforo geralmente é muito baixo ${ }^{20}$. Os conteúdos individuais dos diferentes elementos nas $\mathrm{SH}$, assim como as relações entre eles, dependem da fonte de matéria orgânica, do grau de humificação e das condições ambientais.

A Tabela 1 mostra a composição elementar dos AH estudados. Os valores aqui medidos encontram-se, em geral, dentro da faixa daqueles encontrados na literatura ${ }^{21}$.

Tabela 1. Análise elementar dos ácidos húmicos estudados

\begin{tabular}{ccccccc}
\hline Amostra & $\mathrm{C}(\%)$ & $\mathrm{H}(\%)$ & $\mathrm{N}(\%)$ & $\mathrm{S}(\%)$ & $\mathrm{C} / \mathrm{H}^{\mathrm{a}}$ & $\mathrm{C} / \mathrm{N}^{\mathrm{b}}$ \\
\hline Aldrich & 51,6 & 4,8 & 1,0 & 1,1 & 0,89 & 60,2 \\
AH1 & 48,7 & 5,3 & 3,2 & 2,3 & 0,77 & 17,7 \\
ITA3 & 51,4 & 6,2 & 4,3 & nd $^{\mathrm{c}}$ & 0,69 & 13,9 \\
CLA1 & 35,3 & 4,4 & 2,7 & 1,6 & 0,67 & 15,3 \\
Gran2 & 31,6 & 4,0 & 3,2 & 1,4 & 0,66 & 11,6 \\
\hline
\end{tabular}

${ }^{\mathrm{a}} \mathrm{C} / \mathrm{H}=(\% \mathrm{C} / 12,01) /(\% \mathrm{H} / 1,00)$

${ }^{\mathrm{b}} \mathrm{C} / \mathrm{N}=(\% \mathrm{C} / 12,01) /(\% \mathrm{~N} / 14,01)$

cnão determinado

As razões atômicas $\mathrm{C} / \mathrm{H}$ e $\mathrm{C} / \mathrm{N}$ têm sido usadas para determinar a fonte de matéria orgânica, o grau de condensação, as transformações diagenéticas e as condições ambientais nas quais os AH se formaram. Sedimentos marinhos, por exemplo, sendo anaeróbios ou menos aerados que solos superficiais e tendo uma fonte de matéria orgânica mais alifática e relativamente rica em nitrogênio, produzem $\mathrm{AH}$ com razões $\mathrm{C} / \mathrm{H}$ e $\mathrm{C} / \mathrm{N}$ mais baixas. Segundo Rice e MacCarthy ${ }^{21}$ o grau de alifaticidade dos $\mathrm{AH}$ decresce de acordo com a ordem: marinho $>$ água doce $>$ solo. Isto acontece porque a matéria orgânica terrígena contém derivados da lignina, presente nas plantas superiores, as quais são predominantes nos ambientes terrestres. Esta tendência é confirmada pelos valores aqui medidos. O AH Aldrich, por exemplo, como representante de origem terrestre apresenta os maiores valores das razões $\mathrm{C} / \mathrm{N}$ e $\mathrm{C} / \mathrm{H}$, indicando um maior grau de insaturação do que nos outros casos. As outras amostras foram obtidas de sedimentos com aportes tanto terrestres quanto aquáticos, marinhos ou não, dependendo do caso. O ácido húmico Gran2, por exemplo, foi extraído de uma amostra de sedimento coletada sob a coluna d'água na desembocadura do Rio Ratones tendo, conseqüentemente, forte predominância da matéria orgânica aquática marinha, o que se reflete nos valores menores das razões C/H e C/N. O ácido húmico AH1, da Lagoa do Peri, também foi extraído de sedimento coletado sob a coluna d'água, entretanto, por ser um lago costeiro de água doce, sem influência marinha, o material aí encontrado tem características próprias. As outras amostras de estuários (ITA3 e CLA1), foram coletadas em zonas inundadas circadianamente por águas marinhas, recebendo ainda material proveniente da vegetação terrestre circundante, ou mesmo de aportes antropogênicos, como no caso da amostra ITA3. Todas estas variáveis contribuem para as pequenas diferenças observadas entre as composições elementares das amostras estudadas.

\section{TS vs. concentração de AH}

A Figura 1 mostra a variação da TS com a concentração para soluções de AH entre 0 e $2000 \mathrm{mg} \mathrm{L}^{-1}$. As medidas foram feitas a $\mathrm{pH}$ 7 visando preservar a relevância ambiental das interpretações e, neste $\mathrm{pH}$, torna-se impossível solubilizar os $\mathrm{AH}$ em concentrações superiores a este valor. Mesmo sendo amostras de origens distintas, os 


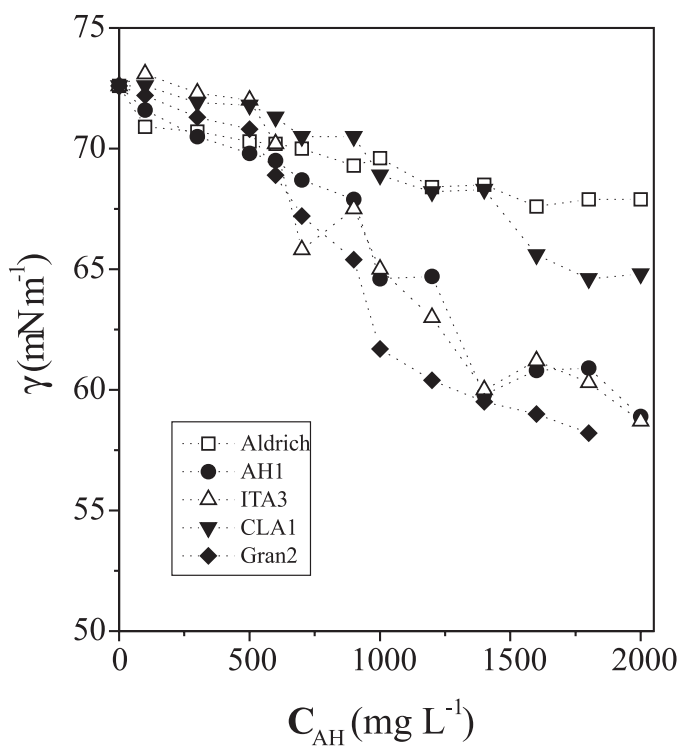

Figura 1. Variação da tensão superficial com a concentração para soluções de ácidos húmicos. $T=22{ }^{\circ} \mathrm{C} ; \mathrm{pH}=7$

AH estudados apresentaram o mesmo padrão de comportamento. Em geral, a TS das soluções diminui sistematicamente com o aumento da concentração de AH até cerca de 1000-1200 mg L $\mathrm{m}^{-1}$, dependendo da amostra. A partir de então a taxa de variação diminui ligeiramente levando a crer que, em alguns casos, alcançaria um valor constante em concentrações maiores. Estes dados reforçam um modelo do tipo anfifílico para os $\mathrm{AH}$, resultante da presença simultânea de grupos hidrófilos e hidrófobos nas estruturas. Na medida em que a concentração de AH aumenta no seio da solução, a presença dos grupos hidrófobos promove a distorção das moléculas de água aumentando a energia livre do sistema. Como forma de minimizar a energia livre e restaurar a estrutura do solvente, as moléculas de $\mathrm{AH}$ migram para a superfície e sua partição na interface ar-água reduz a tensão superficial da solução relativamente à da água pura.

A diminuição na taxa de variação da TS a partir de uma determinada concentração parece indicar que os AH são capazes de se orientar ou se organizar em solução, de modo a proteger suas porções hidrófobas das interações com o solvente, diminuindo assim o percentual de migração para a superfície. Este tipo de arranjo poderia ser intramolecular, considerando-se que os AH são macromoléculas flexíveis que podem enrolar-se sobre si mesmas ${ }^{22}$, ou intermolecular, pela associação de um determinado número de macromoléculas ${ }^{17}$. Tanto num caso como no outro, o interior das aglomerações seria relativamente hidrófobo e o exterior, mais hidrófilo. O termo "pseudomicela" tem sido usado por Wandruszka e colaboradores ${ }^{10-12}$ para definir o primeiro tipo de arranjo.

Os AH de ambientes aquáticos (AH1, Gran2 e ITA3), os quais registraram diminuições de cerca de $22 \%$ com relação ao valor inicial de TS, mostraram-se mais sensíveis do que o AH Aldrich no que se refere à influência da concentração na TS. Esta diferença de resposta pode estar ligada às peculiaridades estruturais entre os dois tipos de material. Duas possibilidades podem ser consideradas para avaliar estas diferenças. Primeiro, um percentual maior de insaturações e conseqüiente hidrofobicidade podem fazer com que a concentração de agregação seja atingida antes, no caso do AH Aldrich. Assim, a pouca variação na TS superficial seria atribuída ao fato do material já estar, desde baixas concentrações formando, em solução, agregados orientados no sentido de proteger seus grupos hidrófobos das interações com o solvente. Wandruszka e colaboradores ${ }^{10-12}$ avaliando as interações entre o pireno e $\mathrm{AH}$ de fontes terrestres têm mostrado que mesmo a $10 \mathrm{mg} \mathrm{L}^{-1}$, em determinadas condições de $\mathrm{pH}$ e força iônica, a agregação intra e/ou intermolecular dos AH pode ocorrer em solução aquosa. Nesta situação a migração para a superfície seria inoperante na redução da energia livre do sistema, o que reduz a influência deste AH na TS da solução. Uma segunda razão, que não descarta a primeira, estaria no tipo e concentração de grupos funcionais ácidos do AH Aldrich. De fato, ácidos húmicos de origem terrestre são, em geral, ricos em grupos fenólicos enquanto os ácidos húmicos de ambientes aquáticos são relativamente mais ricos em grupos carboxílicos ${ }^{20}$. Assim, em pH 7, o grau de protonação é diferenciado nas diversas amostras e deve influenciar no percentual de anfifilicidade e a consequiente capacidade de agregar-se das misturas húmicas. Esta segunda possibilidade será novamente discutida quando da avaliação dos efeitos do $\mathrm{pH}$ e da força iônica.

$\mathrm{O}$ ácido húmico CLA1 tem um comportamento misto entre aquele do AH Aldrich e o das outras três amostras, sendo mais próximo do primeiro. Na realidade, o sedimento utilizado para obtenção desta amostra em particular, apesar de se localizar em um ponto situado à beira da Enseada de Ratones, tem um certo caráter terrestre por estar sob um fragmento de floresta de manguezal, recebendo aportes de água e sedimentos terrestres. Os valores das relações $\mathrm{C} / \mathrm{N}$ e $\mathrm{C} / \mathrm{H}$ para este material sugerem, inclusive, uma estrutura mista, isto é, relativamente pobre em nitrogênio, mas com um grau de saturação tipicamente marinho.

\section{Variação da TS com o pH}

A Figura 2 mostra a variação da TS de soluções de ácidos húmicos com o $\mathrm{pH}$. A concentração de $30 \mathrm{mg} \mathrm{L}^{-1}$ foi escolhida por ser passível de ser encontrada em ambientes aquáticos ricos em matéria orgânica, como é o caso dos estuários ${ }^{23}$.

Em pHs alcalinos, os valores da TS são próximos ou, em alguns casos, ligeiramente maiores do que o da água pura. Com a diminuição do $\mathrm{pH}$ a TS diminui, atingindo, em geral, um mínimo entre $\mathrm{pH}$ 3 e 5. Perfis similares foram obtidos por Yates e Wandruszka ${ }^{17}$, trabalhando com $\mathrm{AH}$ de rio e de solo na concentração de $500 \mathrm{mg} \mathrm{L}^{-1}$. $\mathrm{Na}$ realidade, em condições alcalinas os grupos carboxílicos e

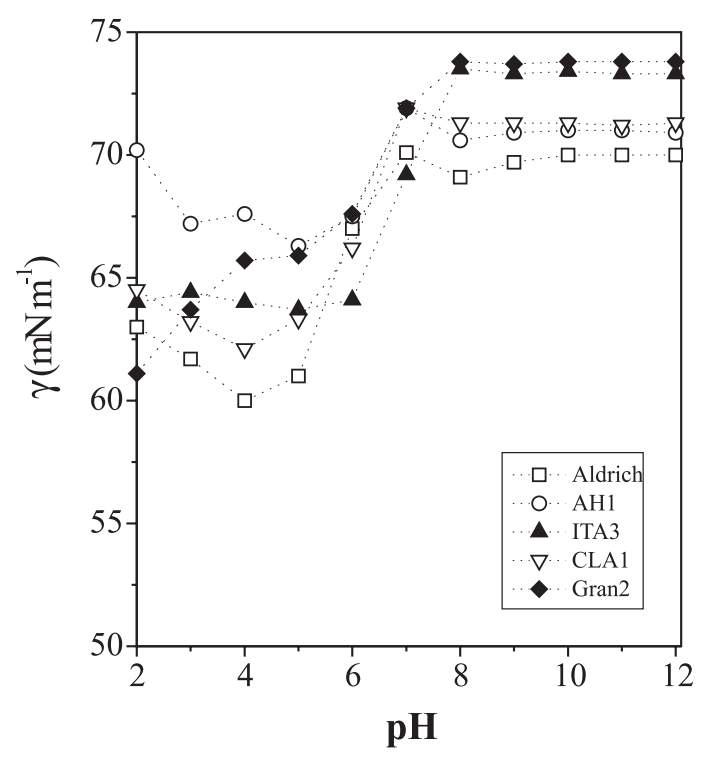

Figura 2. Efeito da variação do pH na tensão superficial de soluções (30 $\mathrm{mg} \mathrm{L}^{-1}$ ) de ácidos húmicos. $\mathrm{T}=22{ }^{\circ} \mathrm{C}$ 
fenólicos dos $\mathrm{AH}$ estão $100 \%$ desprotonados, o que confere às moléculas uma configuração estendida devido à repulsão entre as cargas negativas dos grupos funcionais. Nesta situação de concentração e $\mathrm{pH}$, os $\mathrm{AH}$ devem estar totalmente hidrófilos, não alterando ou mesmo aumentando ligeiramente a TS, ao atrair as moléculas de água para o seio da solução, devido ao aumento da entropia do sistema. Com a diminuição do $\mathrm{pH}$, ocorre a neutralização parcial das cargas negativas pelo próton, o que ocasiona a flexibilização das estruturas húmicas, aumentando o percentual de espécies anfifílicas, as quais migram para a superfície. Este efeito reflete-se na diminuição da TS. Em pHs muito ácidos as moléculas, totalmente neutralizadas, tendem a flocular deixando a superfície, o que ocasiona a restauração parcial do valor da TS, em alguns casos.

Pequenas diferenças de comportamento podem ser observadas se comparamos as amostras entre si. O AH Gran2, por exemplo, não apresenta um valor mínimo de TS nesta faixa de pHs. Outra vez a origem deste material reflete-se no seu comportamento, pois por serem relativamente ricos em grupos carboxílicos e de natureza alifática, com alto grau de saturação, estes compostos são bastante solúveis, não demonstrando sinais de floculação mesmo em pHs ácidos.

É interessante observar que, no que se refere ao efeito do $\mathrm{pH}$, os ácidos húmicos Aldrich e CLA1 mostram tendências similares e com valores mínimos de TS ainda mais baixos do que os das outras amostras. De fato, os AH Aldrich e CLA1 mostram um caráter anfifílico especialmente forte em $\mathrm{pH}$ 4. Assim, a pequena resposta observada no caso do efeito da concentração (Figura 1) parece estar ligada principalmente à concentração relativa e ao tipo de grupos funcionais. Notese que em pH 7 (o pH em que foi analisado o efeito da concentração) o valor da TS do ácido húmico Aldrich ainda é relativamente alto, demonstrando a permanência preferencial das macromoléculas no seio da solução. Somente em pH 6, quando se inicia a protonação dos escassos grupos carboxílicos presentes nas estruturas fazem-se sentir os efeitos da atividade superficial do AH Aldrich. Aliás, em todos os casos estudados, este $\mathrm{pH}$ parece ser crítico no que concerne à atividade superficial dos AH. Pequenas diferenças nas respostas estão ligadas às quantidades relativas de grupamentos ácidos que podem ser neutralizados ou não, em determinados pHs, mudando assim o percentual de estruturas anfifílicas de uma amostra para outra.

\section{Efeito da força iônica}

Para avaliar o efeito de eletrólitos no processo de aglomeração dos AH em solução, medidas da TS em função da concentração foram feitas em presença de $\mathrm{NaCl} 0,3 \mathrm{~mol} \mathrm{~L}^{-1}$. A escolha deste valor de força iônica foi baseada em dois critérios. Primeiro, um teste foi realizado para cada uma das amostras de $\mathrm{AH}$ aqui estudadas, usando-se quantidades crescentes de sal e somente em concentrações superiores a $0,2 \mathrm{~mol} \mathrm{~L}^{-1} \mathrm{de} \mathrm{NaCl}$ algum efeito foi observado. Segundo, um valor de força iônica desta ordem é passível de ser encontrado num ambiente típico estuarino, onde a salinidade pode variar desde 0 até $35 \mathrm{~g} \mathrm{~kg}^{-1}$. Expressa em termos de força iônica esta variação é de 0 a $0,7 \mathrm{~mol} \mathrm{~L}^{-1}$.

A Figura 3 mostra o efeito da variação da força iônica no perfil de TS vs. concentração de $\mathrm{AH}$, para três das amostras: uma estuarina, uma lacustre e para o AH Aldrich. Em cada um dos casos, em maior ou menor grau, o valor de concentração na qual ocorre uma desaceleração da taxa de migração para a superfície diminui consideravelmente quando comparado ao valor das soluções sem adição de sal. $\mathrm{O}$ efeito da adição de $\mathrm{NaCl}$ é, de fato, análogo àquele observado pela diminuição do $\mathrm{pH}$. A neutralização pelo $\mathrm{Na}^{+}$dos grupos hidrofílicos, os quais estavam carregados negativamente neste $\mathrm{pH}$, aumenta o caráter anfifílico dos $\mathrm{AH}$, promovendo sua migração para a superfície, mesmo em concentrações menores. Com a adição de

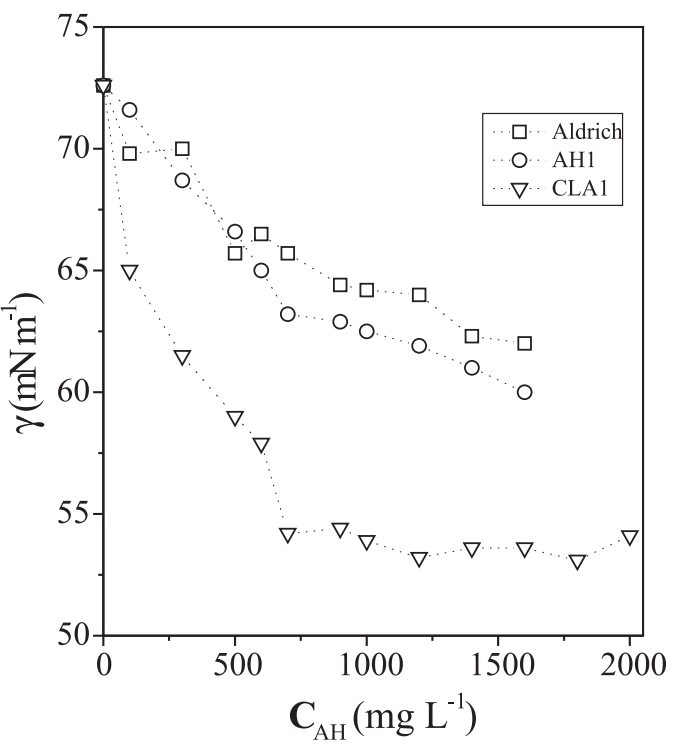

Figura 3. Variação da tensão superficial com a concentração para soluções de ácidos húmicos em presença de $\mathrm{NaCl}(\mathrm{I}=0,3) ; \mathrm{T}=22{ }^{\circ} \mathrm{C} ; \mathrm{pH}=7$

sal é possível igualmente observar com mais nitidez a concentração acima da qual a migração para a superfície cessa. Por meio da interseção das duas regiões lineares, pode-se observar na Figura 4 que este valor fica em torno de $750 \mathrm{mg} \mathrm{L}^{-1}$, para as três amostras. $\mathrm{O}$ modelo micelar parece, efetivamente, ser o mais adequado para descrever este tipo de comportamento. Desta forma, a partir desta concentração crítica de agregação (CCA) que seria correspondente à concentração micelar crítica dos surfactantes, as estruturas húmicas devem rearranjar-se em agregados similares a micelas no seio da solução, cessando a migração para a superfície. Este tipo de interpretação vai ao encontro dos atuais conceitos sobre as estruturas das $\mathrm{SH}$, segundo os quais este material consistiria de moléculas relativamente pequenas e heterogêneas ("building blokcs") ${ }^{24}$ agrupadas em associações supramoleculares ${ }^{25}$.

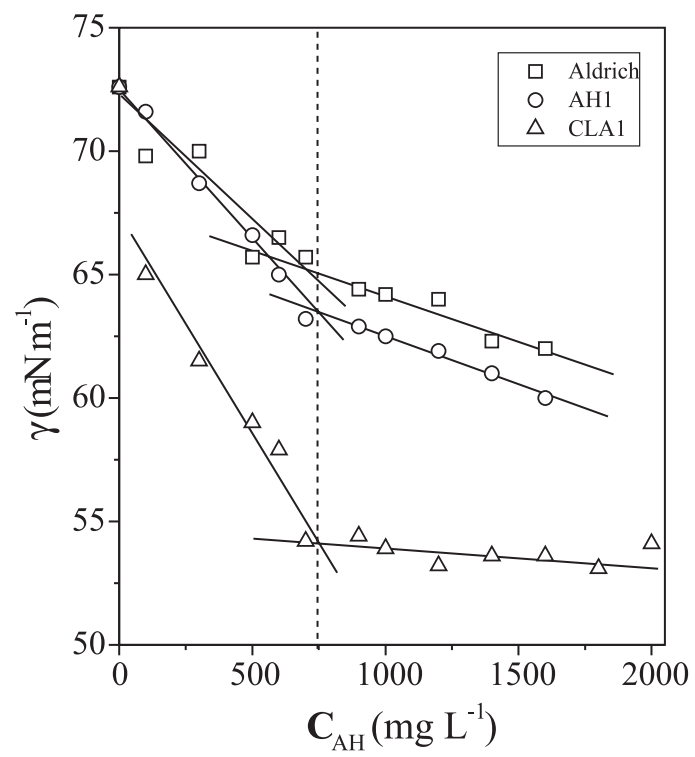

Figura 4. Determinação da concentração crítica de agregação dos compostos húmicos em presença de $\mathrm{NaCl}(\mathrm{I}=0,3) ; \mathrm{T}=22{ }^{\circ} \mathrm{C} ; \mathrm{pH}=7 . \mathrm{O}$ ponto $C=0$ foi ignorado 
Comparando-se as curvas da Figuras 1 e 3 observa-se que o efeito da adição de sal foi mais decisivo na agregação dos ácidos húmicos Aldrich e CLA1 do que no caso do AH1. Este dado vai ao encontro daquele evidenciado pela alteração do $\mathrm{pH}$, ou seja, de que estes dois ácidos húmicos têm, potencialmente, um caráter anfifílico mais forte, provavelmente devido a uma combinação mais balanceada entre hidrofobicidade e hidrofilicidade. Esta anfifilicidade é manifestada, ou não, dependendo do grau de neutralização das cargas negativas, seja pelo próton, seja pela adição de sal. Esta possibilidade de mudança de comportamento, em função das características físico-químicas do ambiente, deve ser considerada quando do estudo das interações entre a matéria orgânica natural e xenobióticos.

Um dado de relevância ambiental que deve igualmente ser enfatizado é que mesmo que as variações na TS ocorram em percentuais diferentes para as diferentes amostras, o valor da CCA é muito semelhante entre elas. Esta uniformidade de resposta, que é igualmente observada nos efeitos da concentração e do $\mathrm{pH}$, mostra que os $\mathrm{AH}$, desde que tenham sido obtidos pelo mesmo processo de extração, apresentam uma identidade comum, independentemente do ambiente onde foram gerados. As implicações deste tipo de observação residem na possibilidade de se elaborar modelos de comportamento que possam ser extrapolados para diferentes situações.

\section{AGRADECIMENTOS}

Os autores agradecem ao CNPq e ao FNMA pelo suporte financeiro assim como aos Profs. V. L. A. F. Bascuñan e D. Zanetti, do Departamento de Química da UFSC, pelas frutíferas discussões dos resultados.

\section{Referências}

1. Lovley, D. R.; Coates, J. D.; Blunt-Harris, E. L.; Philips, E. J. P.; Woodward, J. C.; Nature 1996, 382, 445.
2. Haitzer, M.; Abbt-Braun, G.; Traunspurger, W.; Steinberg, C.E.W.; Environ Toxicol. Chem. 1999, 18, 2782.

3. Kerndorff, H.; Schnitzer, M.; Air and Soil Pollution 1979, 12, 319.

4. Steinberg, C. E. W.; Haitzer, M.; Brüggemann, R.; Perminova, I. V.; Yaschenko, N. Y.; Petrosyan, V. S.; Int. Rev. Hydrobiol. 2000, 85, 253.

5. Rezende, M. O. O.; Resumos do $2^{\circ}$ Encontro Brasileiro Sobre Substâncias Húmicas, São Carlos, Brasil, 1997.

6. Tavares, M. C. H.; Landgraf, M. D.; Vieira E. M.; Rezende, M. O. O.; Quim. Nova 1996, 19, 605.

7. Schnitzer, M.; Khan, S. U.; Humic Substances in the Environment, Marcel Dekker: New York, 1972.

8. Chiou, C. T.; Malcolm, R. L.; Brinton, T. I.; Kile, D. E.; Environ. Sci. Technol. 1986, 502.

9. Engebretson, R. R.; von Wandruszka, R.; Environ. Sci. Technol. 1996, 30, 990.

10. Ragle, C. S.; Engebretson, R. R.; Von Wandruszka, R.; Soil Sci. 1997, 162, 106.

11. Engebretson, R. R.; von Wandruszka, R.; Org. Geochem. 1997, 27, 759.

12. Engebretson, R. R.; von Wandruszka, R.; Environ. Sci. Technol. 1998, 32, 488.

13. Tchapeck, M.; Wasowski, C.; Geochim. Cosmochim. Acta 1976, 40, 1343.

14. Chen, Y.; Schnitzer, M.; Soil Sci. 1978, 125, 7.

15. Hayase, K.; Tsubota, H.; Geochim. Cosmochim. Acta 1983, 47, 947.

16. Anderson, M. A.; Hung, A.; Mills, D.; Scott, M. S.; Soil Sci. 1995, 160, 111.

17. Yates, L. M. III; von Wandruszka, R.; Soil Sci. 1999, 63, 1645.

18. Swift, R. S. Em Methods of Soil Analysis; Soil Science Society of America: Madison, WI, 1996, p. 1018-1020.

19. Adamson, A. W.; Physical Chemistry of Surfaces, $5^{\text {th }}$ ed., Wiley Interscience: Califórnia, 1990.

20. Rashid, M. A.; Geochemistry of Marine Humic Compounds, SpringerVerlag: New York, 1985.

21. Rice, J.A.; MacCarthy, P.; Org. Geochem. 1991, 17, 635.

22. Swift, R. S. Em Humic Substances. II: In search of structure; Hayes, M. H. B; MacCarthy, P.; Malcolm, R. L.; Swift, R. S., eds.; Wiley: Chichester, 1994.

23. Sierra, M. M. D.; Tese de Doutorado, Université de Bordeaux I, França, 1992.

24. Sein Jr., L. T.; Varnum, J. M,; Jansen, S. A.; Environ. Sci. Technol. 1999, 33, 546.

25. Cozzolino, A.; Conte, P.; Piccolo, A.; Soil Biol. Biochem. 2001, 33, 563. 